\title{
Cysteinyl-glycine reduces mucosal proinflammatory cytokine response to fMLP in a parenterally-fed piglet model
}

\author{
Matthew G. Nosworthy' and Janet A. Brunton'
}

BACKGROUND: PepT1 transports dietary and bacterial peptides in the gut. We hypothesized that cysteinyl-glycine would ameliorate the inflammatory effect of a bacterial peptide, formylmethionyl-leucyl-phenylalanine (fMLP), in both sow-fed and parenterally-fed piglets.

METHODS: An intestinal perfusion experiment was performed in piglets $(N=12)$ that were sow-reared or provided with parenteral nutrition (PN) for $4 \mathrm{~d}$. In each piglet, five segments of isolated intestine were perfused with five treatments including cysteine and glycine, cysteinyl-glycine, fMLP, free cysteine and glycine with $\mathrm{FMLP}$, or cysteinyl-glycine with $\mathrm{fMLP}$. Mucosal cytokine responses and intestinal morphology was assessed in each gut segment.

RESULTS: PN piglets had lower mucosal IL-10 by approximately 20\% ( $P<0.01)$. Cysteinyl-glycine lowered TNF- $\alpha$ response to $f M L P$ in $P N$-fed animals and IFN- $\gamma$ response to fMLP in both groups $(P<0.05)$. The free cysteine and glycine treatment reduced TNF- $\alpha$ in sow-fed animals $(P<0.05)$. fMLP affected villus height in parenterally $(P<0.05)$, but not sow-fed animals.

CONCLUSION: Parenteral feeding conferred a susceptibility to mucosal damage by fMLP. The dipeptide was more effective at attenuating the inflammatory response to a bacterial peptide than free amino acids. This may be due to competitive inhibition of fMLP transport or a greater efficiency of transport of dipeptides.

$\mathbf{T}$ he products of protein digestion that are absorbed by the small intestinal epithelium include free amino acids and small peptides of two to three residues in length. These di/tripeptides are removed from the nutrient rich intestinal lumen by a $\mathrm{H}^{+} /$peptide symporter, peptide transporter 1 or PepT1 (1). This transporter is localized to the apical surface of the intestinal villi and has broad substrate specificity. Potential substrates for PepT1 include almost all possible dietary di/ tripeptides (1), some antibiotics (2-4) and proinflammatory bacterial peptides (5-8).

The primary neutrophil chemotactic substance produced by Escherichia coli is formyl-methionyl-leucyl-phenylalanine (fMLP) (9). This tripeptide is the most predominant $\mathrm{N}$-formylated peptide present in the colonic lumen of humans (9).
PepT1-mediated transport of fMLP has been demonstrated in cell culture where uptake of fMLP was inhibited by the presence of known substrates of PepT1 $(10,11)$. Further, the presence of fMLP induced neutrophil migration across an epithelial monolayer, an activity that was abolished if fMLP uptake was inhibited. PepT1-mediated transport of fMLP has also been demonstrated using in vivo rat models $(12,13)$. In particular, uptake of fMLP has been investigated in rats using an intestinal perfusion approach (12). Marked inflammatory response occurred in jejunal segments perfused with fMLP, an intestinal position known to have high expression of PepT1. This inflammation was accompanied by an increase in DNA binding by $\mathrm{NF \kappa} \beta$, a transcription factor involved in the regulation of proinflammatory cytokines. This transcription factor is capable of inducing transcription of TNF- $\alpha$, a potent proinflammatory cytokine involved in inflammatory disease (14). Similarly, NFK $\beta$ is capable of promoting the transcription of IFN- $\gamma$ (15), another proinflammatory cytokine. Therefore the induction of NFK $\beta$ by fMLP may also increase the concentration of both IFN- $\gamma$ and TNF- $\alpha$. Competitive inhibition of fMLP transport, or direct regulation of PepT1 expression, may have the potential to ameliorate intestinal inflammation in pathological conditions that result in an abnormally high presence of bacterial peptides in the small intestine.

The necessity for parenteral nutrition (PN) support represents a pathological situation for the gut that is characterized by greater intestinal permeability with potential for intestinal atrophy (16). Furthermore, PN modulates the immune response of the intestine leading to higher risk of infection $(17,18)$. This risk is potentiated via the suppression of the bactericidal response of the small intestine (17) and a reduction in Paneth cell function leading to an inability to replenish lost enterocytes (18). A study measuring mRNA of amino acid transporters of parenterally and orally fed adult rats noted an increase in PepT1 mRNA in the distal small intestine (19). This combination of sustained PepT1 with bacterial overgrowth in the small intestine, or bacterial infection due to $\mathrm{PN}$, may facilitate greater uptake of bacterial peptides leading to the development of intestinal inflammation.

We used a piglet model of $\mathrm{PN}$ in combination with a ligated loop model of intestinal perfusion to investigate differences in cytokine concentrations and intestinal morphology after perfusion with fMLP alone or in combination with a competitor 


\section{Articles | Nosworthy and Brunton}

for PepT1, the dipeptide cysteinyl-glycine. The purpose of this study was to quantify factors related to an inflammatory response to fMLP in the distal small intestine of piglets with $\mathrm{PN}$-induced intestinal changes, compared to healthy sowfed littermates. Further, we determined whether the mucosal response to fMLP was altered when presented to the small intestine with a dipeptide (cysteinyl-glycine), or the constituent free amino acids (L-cysteine and glycine). The selection of cysteinyl-glycine as the dipeptide for this study was based on the anti-inflammatory capacity of cysteine (20) as well as our previous work demonstrating that cysteinyl-glycine reduced the mucosal concentration of proinflammatory cytokines in a piglet model of short bowel syndrome (21)

\section{RESULTS}

Throughout the perfusion studies, all piglets remained stable, well-oxygenated, and maintained a core body temperature between $37-39{ }^{\circ} \mathrm{C}$. Piglet weights did not differ between the two diet groups at the initiation of the loop procedure (PN: $2.60 \pm 0.23$, SF: $2.61 \pm 0.23$ ).

\section{Mucosal Cytokines}

Compared to SF controls, PN did not affect mucosal IFN- $\gamma$ concentrations in intestinal loops not exposed to fMLP (Figure 1a). Perfusion of intestinal loops with fMLP alone significantly increased the concentration of IFN- $\gamma$ in both feeding groups $(P<0.001)$. Coperfusion of fMLP with cysteinyl-glycine resulted in significantly lower concentrations of IFN- $\gamma$ in PN-fed piglets only $(P<0.01)$. In sow-fed piglets, inclusion of cysteinyl-glycine resulted in a variable IFN- $\gamma$ response that was not significantly different from the control or fMLP loops. No significant differences were measured when fMLP was coperfused with free cysteine and glycine. Similar to the IFN- $\gamma$ response, there was no effect of PN on mucosal TNF- $\alpha$ concentrations compared to $\mathrm{SF}$ in unstimulated gut segments (Figure 1b). In both feeding methods, perfusion of fMLP resulted in a greater concentration of TNF- $\alpha$ compared to the control loops, regardless of the presence of either cysteine + glycine or cysteinyl-glycine $(P<0.05)$. Treatment with fMLP and amino acids demonstrated that there was a significant effect of the form of amino acids. Compared to fMLP alone, the dipeptide resulted in lower TNF- $\alpha$ concentrations stimulated by fMLP in both sow-fed and parenterallyfed piglets $(P<0.05)$. Overall, SF piglets had lower TNF- $\alpha$ responses to fMLP compared to PN animals when either free cysteine and glycine or the dipeptide was added to the perfusate $(P<0.01)$. The mucosal concentration of IL-10 was not affected by any loop treatment; however, piglets receiving PN had lower concentrations of the regulatory cytokine than their sow-fed littermates $(P<0.01)$ (Figure 1c).

\section{Disappearance of ${ }^{3} \mathrm{H}$-fMLP or ${ }^{14} \mathrm{C}$-Mannitol}

Perfused buffers sampled throughout the 3 -h procedure were analyzed to determine disappearance of ${ }^{3} \mathrm{H}$-fMLP, as a marker for fMLP transport and ${ }^{14} \mathrm{C}$-mannitol, an indicator of paracellular transport. Scintillation counting of the perfusate samples revealed highly variable results for the transport of ${ }^{3} \mathrm{H}$-fMLP in

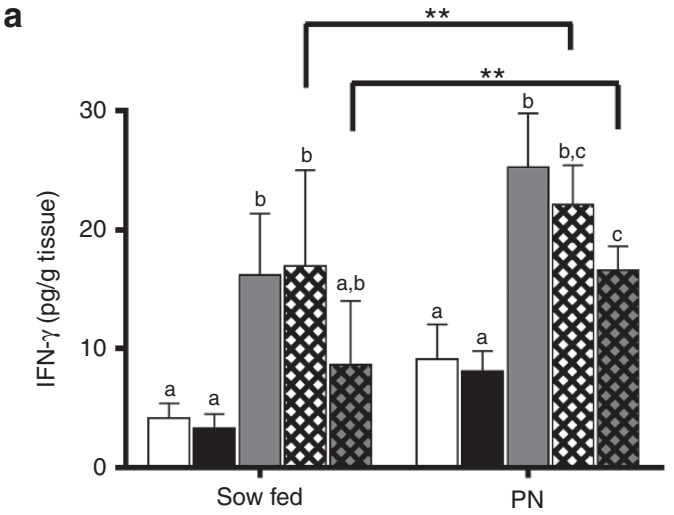

b

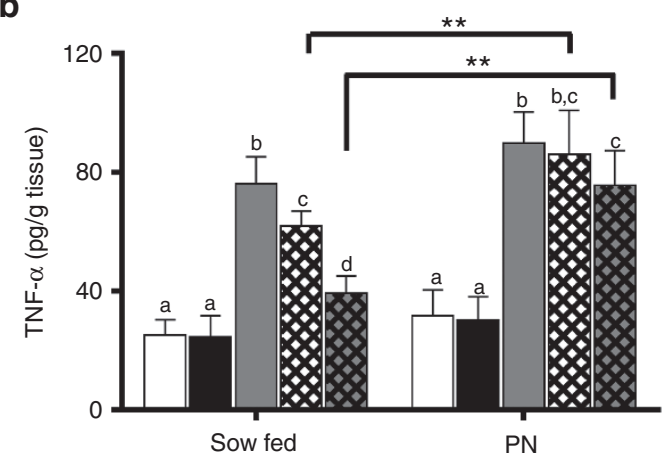

c

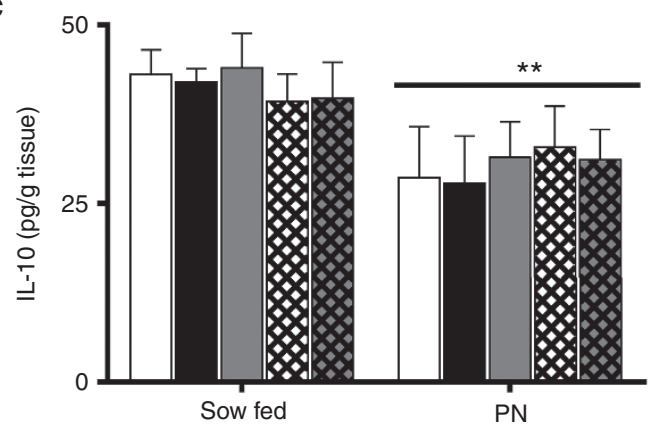

Figure 1. Effect of fMLP and cysteinyl-glycine on mucosal cytokine concentrations. Mucosal cytokine concentrations, (a) IFN- $\gamma$, (b) TNF- $\alpha$, and (c) IL-10, in sow-fed and parenteral nutrition-fed piglets sampled from ligated loops perfused with cysteine + glycine (white), cysteinyl-glycine (black), formyl-methionyl-leucyl-phenylalanine (gray), a combination of cysteine + glycine + formyl-methionyl-leucyl-phenylalanine (fMLP) (white hatched) or cysteinyl-glycine + fMLP (gray hatched). Lines represent significant differences between diet treatments $\left({ }^{*} P<0.01\right)$. Differing letters indicate differences amongst loop treatments within the diet treatment $(P<0.05)$. Data were analyzed via mixed model two-way ANOVA with Bonferroni post-hoc analysis. $N=6$ per group. Values are mean $+S D$.

all loops, both in PN and SF piglets, with no significant differences found either between dietary regimen or gut loop treatments (data not shown). Analysis of ${ }^{14} \mathrm{C}$-mannitol disappearance showed no differences in mannitol concentration with any treatment or in either sow-fed or PN fed piglets (data not shown).

\section{Myeloperoxidase Activity}

Myeloperoxidase activity was measured in the intestinal mucosa as an indicator of neutrophil migration. There was no effect of route of feeding on basal myeloperoxidase (MPO) activity 


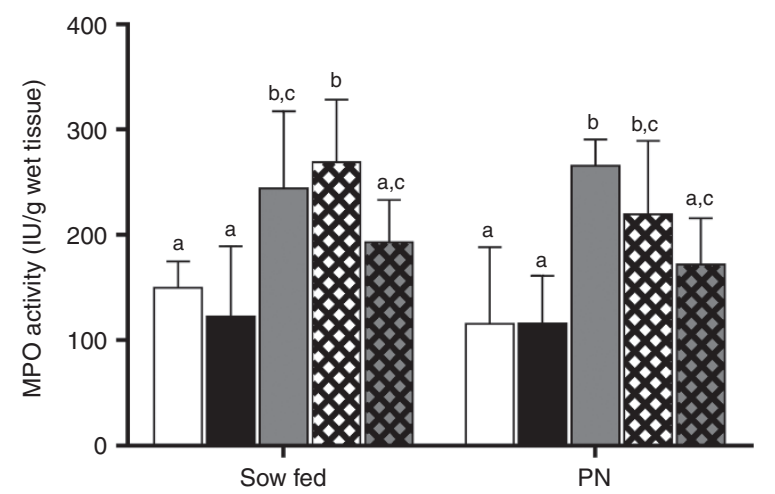

Figure 2. Mucosal myeloperoxidase activity in ileal mucosa of sow-fed and parenteral nutrition-fed piglets sampled from ligated loops perfused with cysteine + glycine (white), cysteinyl-glycine (black), formyl-methionyl-leucyl-phenylalanine (gray), a combination of cysteine + glycine + formyl-methionyl-leucyl-phenylalanine (fMLP) (white hatched) or cysteinyl-glycine $+\mathrm{fMLP}$ (gray hatched). Differing letters represent significant differences between loop treatments within a feeding group $(P<0.05)$. Data were analyzed via mixed model two-way ANOVA with Bonferroni post-hoc test. $N=6$ per group. Values are mean $+S D$.

(Figure 2). Perfusion of intestinal segments with fMLP generated greater MPO activity in both groups, regardless of dietary regimen $(P<0.05)$. While the inclusion of cysteine + glycine with $\mathrm{AMLP}$ had no impact on MPO activity, samples coperfused with cysteinyl-glycine resulted in MPO activity that was similar to that of the control loops in both SF and PN piglets.

\section{Intestinal Morphology}

There was no effect of route of feeding or loop treatment on crypt depth (Figure 3). In the sow-fed animals, villus height was unaffected by exposure to fMLP. In the PN group, however, villi were significantly shorter in the loops perfused with fMLP $(P<0.05)$, and co-perfusion with either cysteine and glycine or cysteinyl-glycine were intermediate between the control and fMLP-treated gut segments.

\section{DISCUSSION}

The objectives of this study were to investigate the mucosal response to fMLP-induced inflammation in healthy vs. compromised gut and to determine the impact of cysteinyl-glycine on fMLP-induced inflammation. Not surprisingly, we determined that parenterally-fed animals were more susceptible to fMLP-induced inflammation than their sow-fed littermates. A novel finding is that cysteinyl-glycine was more effective at attenuating fMLP-induced TNF- $\alpha$ production in sow fed animals, compared to equimolar amounts of the constituent free amino acids.

Intestinal mucosa exposed to only fMLP had greater concentrations of IFN- $\gamma$ and TNF- $\alpha$ compared to control conditions. Previous work on other cell types such as neurons (22), myeloid cells, (23) and peripheral blood monocytes (24) demonstrated that fMLP exposure induces expression of NF- $\kappa \beta$. In turn, NF- $\kappa \beta$ can act on the promoter regions for proinflammatory cytokines such as IFN- $\gamma$ and TNF- $\alpha$ (14). In a positive feedback loop, TNF- $\alpha$ is also capable of inducing NF- $\kappa \beta$

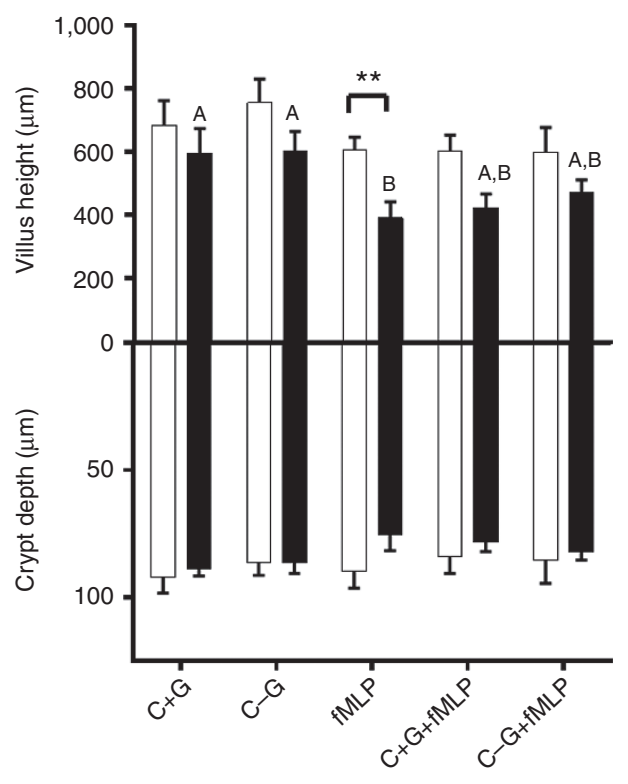

Figure 3. Villus height $(>0)$ and crypt depth $(<0)$ in the ileum of sow-fed (white)and parenteral nutrition-fed (black) piglets sampled from ligated loops perfused with cysteine + glycine $(C+G)$, cysteinyl-glycine $(C-G)$, formyl-methionyl-leucyl-phenylalanine (fMLP), a combination of cysteine + glycine + fMLP (C+G+fMLP) or cysteinyl-glycine + fMLP (C-G+fMLP). Differing letters represent significant differences between loop treatments within parenterally fed animals $(P<0.05)$. Lines represent significant differences between diet treatment $\left({ }^{*} P<0.05\right)$. Data were analyzed via mixed model two-way ANOVA with Bonferroni post-hoc test. $N=6$ per group. Values are mean \pm SEM.

activation resulting in the propagation of the inflammatory response (25). Although we did not directly measure NF- $\kappa \beta$, the conserved nature of the response to fMLP exposure across numerous cell types combined with the fMLP-induced increase of proinflammatory cytokines in the intestine suggests that enterocytes respond to fMLP in a similar manner.

Inclusion of the dipeptide cysteinyl-glycine attenuated fMLP-induced production of IFN- $\gamma$ and TNF- $\alpha$, potentially through the reduction of PepT1-mediated fMLP uptake via competitive inhibition. fMLP transport inhibition in the presence of other PepT1 substrates has been well described $(5,11,12)$. In this study, exposure of gut segments to fMLP resulted in greater concentrations of proinflammatory cytokines compared to control conditions, while coperfusion with cysteinyl-glycine attenuated this response as demonstrated by lower mucosal TNF- $\alpha$ concentrations. Lower tissue concentration of TNF- $\alpha$ potentially has a dual effect on the immune response; firstly, there would be a direct reduction of inflammation because of lower exposure to TNF- $\alpha$ and also, the autostimulation of TNF- $\alpha$ production through NF- $\kappa \beta$ would be suppressed $(14,25)$. Thus, the intentional provision of high concentrations of PepT1 substrates may lead to further reduction in the inflammatory response.

Interestingly, gut segments exposed to fMLP in combination with free cysteine and glycine suppressed the TNF- $\alpha$ response in sow-fed piglets, but not in the PN-fed littermates. Cysteine is part of the oxidative stress control system, independent of its role as a component of glutathione (20). Both cysteine and 
glycine have been demonstrated to exhibit anti-inflammatory effects in arterial endothelial cells through the reduction of NF- $\kappa \beta$ activation (26). The differing responses by diet treatment may be related to impairment of free amino acid uptake secondary to $\mathrm{PN}$-induced intestinal atrophy (27); thus, the tempered response to free cysteine and glycine could be due to reduced amino acid availability.

To determine whether regulatory cytokines were also affected by route of feeding or fMLP exposure, we quantified mucosal concentration of interleukin-10 (IL-10). Unlike the proinflammatory cytokines measured, IL-10 concentration was not altered by exposure to fMLP. Piglets receiving PN, however, had lower mucosal IL-10 compared to their sow-fed littermates. Parenteral nutrition has been documented to lower IL-10 concentration $(28,29)$, and to increase intestinal permeability (30-32); however, no difference in ileal permeability was detected in this study. As IL-10 can suppress IFN- $\gamma$ production (33), the lower IL-10 concentrations in PN-fed animals could have contributed to the higher concentrations of IFN- $\gamma$ found in this piglets when compared to SF littermates.

The disappearance of fMLP using a radiolabelled substrate was also quantified in this study, but we found no significant differences among any of the loop treatments or between dietary treatments. There are two possible explanations for this result. It may be that there was no difference in the uptake of fMLP from the perfusate. Alternatively, it is possible that the method we used to quantify fMLP disappearance was not sufficiently sensitive. Indirect evidence of fMLP uptake was provided by the differing concentrations of mucosal cytokines in the presence of the bacterial peptide. Similarly, different mucosal responses to fMLP perfused alone, with free amino acids or with cysteinyl-glycine suggest that there was potential inhibition when a dipeptide was present. Thus, we speculate that the detection method was not sensitive enough to quantify the movement of ${ }^{3} \mathrm{H}$-fMLP. As a surrogate indicator of fMLP transport and its resultant impact on intestinal inflammation, mucosal myeloperoxidase activity was measured.

MPO activity was not affected by intestinal atrophy induced by $\mathrm{PN}$, and cysteinyl-glycine was effective at attenuating fMLP-induced MPO activity in both sow-fed and PN animals. Previous studies have investigated MPO activity in response to fMLP in the small intestine and colon of rats $(5,12)$. These investigations demonstrated that exposure to fMLP stimulated MPO activity in the small intestine, and only in colonic tissue of rats with PepT1 expression induced by small bowel resection. We have demonstrated that supplying a competitive inhibitor for fMLP uptake, cysteinyl-glycine, prevents significant stimulatory effects of fMLP on MPO activity in parenterallyfed piglets. Many studies have shown competitive inhibition of fMLP transport with dipeptides or other substrates of PepT1 $(5,11,12)$, but whether there is an additional benefit due to the presence of a particular dipeptide has yet to be determined.

The architecture of the ileal mucosa was not significantly altered after $4 \mathrm{~d}$ of parenteral feeding, although PN-feeding increased its susceptibility to fMLP-induced villus damage. Remarkably, after only $180 \mathrm{~min}$ of exposure to fMLP, villus height was measurably reduced. This effect is not limited to the ileum; a study in rats investigated the inflammatory effect of fMLP and found significant villus damage in the jejunum after perfusion with fMLP (12). In the current study, the villi damage induced by fMLP was partially avoided when either cysteine + glycine or cysteinyl-glycine was included in the perfusate.

This study was the first to investigate the consequences of fMLP-induced inflammation in a parenterally-fed animal model. We found that parenteral feeding led to greater indices of fMLP-induced inflammation in the ileum and that the inclusion of a dipeptide, cysteinyl-glycine, in the lumen of the intestine partially ameliorated this response. Thus, deliberate feeding of peptides may represent an important therapeutic strategy toward reducing intestinal inflammation in fragile neonates in high risk situations, such as during the reintroduction of enteral feeding. Further investigation into the potential benefits of specific peptides with anti-inflammatory or antioxidatitive activities is warranted.

\section{METHODS}

\section{Study Design}

All experimental procedures were approved by the Institutional Animal Care Committee in accordance with guidelines of the Canadian Council of Animal Care. Yucatan miniature piglets were randomized to either parenteral nutrition $(\mathrm{PN}, N=6)$ or sow-feeding $(N=6)$ as littermate pairs. The littermates assigned to $\mathrm{PN}$ were removed from the sow at $10 \mathrm{~d}$ of age and underwent surgical insertion of jugular and femoral catheters. The catheters were implanted for blood sampling and delivery of PN. PN was initiated immediately following surgery and continued until day 4 postoperatively. The complete PN diet provided 1.1 MJ of metabolizable energy $\cdot \mathrm{kg}^{-1} \cdot \mathrm{d}^{-1}$ with glucose $\left(24.5 \mathrm{~g} \cdot \mathrm{kg}^{-1} \cdot \mathrm{d}^{-1}\right)$ and lipid (20\% Intralipid, Pharmacia) each supplying $50 \%$ of nonprotein energy and $15 \mathrm{~g} \cdot \mathrm{kg}^{-1} \cdot \mathrm{d}^{-1}$ of protein, supplied as free amino acids. The amino acid composition was as follows (per gram of total L-amino acids): alanine, $107 \mathrm{mg}$; arginine, $67 \mathrm{mg}$; aspartate, $61 \mathrm{mg}$; cysteine, $14 \mathrm{mg}$; glutamate, $105 \mathrm{mg}$; glycine, $27 \mathrm{mg}$; histidine, $31 \mathrm{mg}$; isoleucine, $46 \mathrm{mg}$; leucine, $104 \mathrm{mg}$; lysine$\mathrm{HCl}, 102 \mathrm{mg}$; methionine, $19 \mathrm{mg}$; phenylalanine, $55 \mathrm{mg}$; proline, $83 \mathrm{mg}$; serine, $56 \mathrm{mg}$; taurine, $5 \mathrm{mg}$; threonine, $41 \mathrm{mg}$; tryptophan, $21 \mathrm{mg}$; tyrosine, $8 \mathrm{mg}$; and valine, $53 \mathrm{mg}$ (34). Prior to feeding, vitamins (Multi-12K1 Pediatric, Sabex, St Boucherville, QC), trace minerals at $200 \%$ of National Research Council recommendations, (NRC, 1998), lipid, and iron dextran (Fe, $3.0 \mathrm{mg} / \mathrm{kg}$; Vetoquinol Canada, Saint-Hyacinthe, QC) were added to the diet.

\section{In Situ Perfusion (Gut Loop Model)}

On day 4 of study (d4 post-op for PN piglets), sow-fed and PN piglets were brought to the laboratory to undergo an in situ perfusion study. The piglets were preanesthetized with an IM injection of ketamine $(20 \mathrm{mg} / \mathrm{kg})$ plus acepromazine $(0.5 \mathrm{mg} / \mathrm{kg})$. Subsequently, the piglets were intubated and maintained under general anesthesia using 0.6$1.0 \%$ isoflurane (Abbott Laboratories, Montreal, QC) mixed with oxygen at a flow rate of $1.5 \mathrm{l} / \mathrm{min}$. A laparotomy was performed to expose the small intestine. The sites for the five intestinal loops were located along the length of the distal small intestine (i.e., ileum). Closed loops of intestine consisted of $10 \mathrm{~cm}$ sections of intestine with inlet and outlet cannulas (inner diameter, $0.2 \mathrm{~cm}$; outer diameter, $0.3 \mathrm{~cm}$, Watson Marlow Pumps Group, Wilmington, MA) inserted through a small perforation at both ends of the $10 \mathrm{~cm}$. A suture was placed around the tube and intestine, occluding flow of intestinal contents into that section. The loop was gently flushed of luminal contents using warmed $\left(37^{\circ} \mathrm{C}\right)$ Krebs-Ringer buffer until the effluent ran clear. Loops were separated by $30 \mathrm{~cm}$ of intestine with the last loop being placed $50 \mathrm{~cm}$ from the ileocecal valve. Piglets were kept warm by a homeothermic blanket and the exposed intestines were kept moistened with 
warmed saline and covered with gauze and plastic wrap. Heart rate, body temperature, and blood oxygenation were monitored throughout the 180 min perfusion, after which the loops were excised by cautery and flushed with cold $0.9 \%$ saline. A 2 -cm segment of loop tissue was immersed in neutral buffered 10\% formalin (Fisher Scientific, Pittsburgh, PA) for histologic analyses. The remaining segment was cut longitudinally, placed on a chilled glass plate and scraped with a microscope slide to remove the mucosa which was then frozen in liquid nitrogen and stored at $-80^{\circ} \mathrm{C}$ for further analysis.

\section{Perfusates}

Five different loop treatments (perfusates) were randomly assigned to intestinal position with treatments matched for location between littermates. The treatments contained: (i) $5 \mathrm{mmol} / \mathrm{l}$ cysteine + glycine (Sigma Aldrich, Oakville, ON); (ii) $5 \mathrm{mmol} / \mathrm{l}$ cysteinyl-glycine (Bachem, Torrence, CA); (iii) $10 \mu \mathrm{mol} / \mathrm{l} \mathrm{fMLP}$ (Sigma Aldrich); (iv) $5 \mathrm{mmol} / \mathrm{l}$ cysteine + glycine $+10 \mu \mathrm{mol} / \mathrm{l} \mathrm{fMLP;} \mathrm{(v)} 5 \mathrm{mmol} / \mathrm{l}$ cysteinyl-glycine $+10 \mu \mathrm{mol} / \mathrm{l} \mathrm{fMLP}$. The fMLP containing perfusates also included ${ }^{3} \mathrm{H}$-fMLP (Moravek, Brea, CA) and all loops contained ${ }^{14} \mathrm{C}$-mannitol (Moravek) to assess intestinal permeability.

\section{Disappearance of ${ }^{3} \mathrm{H}$-fMLP and ${ }^{14} \mathrm{C}$-Mannitol}

Sampled perfusate $(100 \mu \mathrm{l})$ was added to $4 \mathrm{ml}$ Scintiverse (Fisher Scientific) for liquid scintillation counting. The specific radioactivity was calculated as the mean dpm/mmol of ${ }^{3} \mathrm{H}$-fMLP or ${ }^{14} \mathrm{C}$-mannitol present in the perfusate and was then used to determine the total quantity of fMLP or mannitol remaining in the perfusates at the time points studied.

\section{Cytokine Analysis}

Mucosal TNF- $\alpha$, IFN- $\gamma$, and IL-10 concentrations were determined via porcine ELISA kits (Pierce, Rockford, IL). Tissue supernatants were prepared by homogenizing tissue in PBS containing Protease Inhibitor Cocktail III (Calbiochem, Etobicoke, ON) and $1 \mathrm{mmol} / \mathrm{l}$ PMSF (Sigma Aldrich). Homogenates were then centrifuged at > $10,000 \mathrm{~g}$ for $5 \mathrm{~min}$ at $4{ }^{\circ} \mathrm{C}$ to allow for analysis of tissue supernatants according to the protocol provided by the supplier. Linear regression was used to calculate the final concentration of cytokine in the supernatant which was reported as pg per gram of mucosa.

\section{MPO Assay}

Mucosal samples (50-100 mg) were homogenized on ice in $0.5 \%$ hexadecyltrimethylammonium bromide (Sigma Aldrich) in $50 \mathrm{mmol} / \mathrm{l}$ $\mathrm{KPO}_{4}$. Homogenates underwent three rapid freeze/thaw cycles $\left(-80^{{ }^{\circ}} \mathrm{C} / 37^{\circ} \mathrm{C}\right)$ and were then centrifuged at $12,800 \mathrm{~g}$ for $15 \mathrm{~min}$ at $4{ }^{\circ} \mathrm{C}$. The supernatant containing MPO was assayed spectrophotometrically after the addition of $50 \mathrm{mmol} / \mathrm{KPO}_{4}$ containing $0.53 \mathrm{mmol} / \mathrm{l}$ $\mathrm{O}$-dianisidine dihydrochloride and $0.15 \mathrm{mmol} / \mathrm{l}$ hydrogen peroxide. Changes in absorbance were measured at $460 \mathrm{~nm}$ for $2 \mathrm{~min}$ with readings taken every $15 \mathrm{~s}$ (BU-530, Beckman Coulter, Mississauga, ON). MPO activity was reported as IU/g wet tissue where one IU was defined as the quantity of enzyme able to convert $1 \mu$ mol of hydrogen peroxide to water in $1 \mathrm{~min}$ at room temperature.

\section{Histological Analysis}

Preparation of slides. After fixation in 10\% buffered formalin (Fisher Scientific), samples of intestine were dehydrated in ethanol, cleared in xylene, embedded in paraffin wax, and sliced into 5- $\mu \mathrm{m}$ sections.

\section{Crypt Depth/Villus Height}

Sections were stained with hematoxylin and eosin (Fisher Scientific). Villus height and crypt depth were measured with a Zeiss Axiostar microscope (Carl Zeiss, Toronto, ON). Images were captured with an Infinity 1 camera and Infinity Analyze software (Lumenera Corporation, Nepean, ON). Ten measurements of villus height and crypt depth were performed per sample. All histological measurements were performed in a blinded manner by a single investigator (M.G.N.).

\section{Statistical Analysis}

Data are presented as means and SDs. For all analyses, a mixed model two-way ANOVA with Bonferroni post-hoc analysis was used, with loop treatment as the repeated measure within pigs and diet as the second variable. Piglets receiving parenteral feeding were matched to sow-fed littermates of the same gender. Differences were determined to be significant if $P<0.05$ (Graphpad Prism 5.0, La Jolla, CA)

\section{STATEMENT OF FINANCIAL SUPPORT}

This study was supported by Canadian Institutes of Health Research (Funding Reference Number 135871) and Research and Development Corporation of Newfoundland (Leverage R\&D 5404.1187.103).

Disclosures: The authors have no conflicts of interest to disclose.

\section{REFERENCES}

1. Vig BS, Stouch TR, Timoszyk JK, et al. Human PEPT1 pharmacophore distinguishes between dipeptide transport and binding. J Med Chem 2006;49:3636-44.

2. Terada T, Saito H, Mukai M, Inui K. Recognition of beta-lactam antibiotics by rat peptide transporters, PEPT1 and PEPT2, in LLC-PK1 cells. Am J Physiol 1997;273(5 Pt 2):F706-11.

3. Terada T, Saito H, Inui K. Interaction of beta-lactam antibiotics with histidine residue of rat $\mathrm{H}+$ /peptide cotransporters, PEPT1 and PEPT2. J Biol Chem 1998;273:5582-5.

4. Zhang Q, Liu Q, Wu J, et al. PEPT1 involved in the uptake and transepithelial transport of cefditoren in vivo and in vitro. Eur J Pharmacol 2009;612:9-14.

5. Shi B, Song D, Xue H, Li N, Li J. PepT1 mediates colon damage by transporting fMLP in rats with bowel resection. J Surg Res 2006;136:38-44.

6. Carlson RM, Vavricka SR, Eloranta JJ, et al. fMLP induces Hsp27 expression, attenuates NF-kappaB activation, and confers intestinal epithelial cell protection. Am J Physiol Gastrointest Liver Physiol 2007;292:G1070-8.

7. Buyse M, Berlioz F, Guilmeau S, et al. PepT1-mediated epithelial transport of dipeptides and cephalexin is enhanced by luminal leptin in the small intestine. J Clin Invest 2001;108:1483-94.

8. Dalmasso G, Nguyen HT, Charrier-Hisamuddin L, et al. PepT1 mediates transport of the proinflammatory bacterial tripeptide L-Ala-\{gamma\}D-Glu-meso-DAP in intestinal epithelial cells. Am J Physiol Gastrointest Liver Physiol 2010;299:G687-96.

9. Marasco WA, Phan SH, Krutzsch H, et al. Purification and identification of formyl-methionyl-leucyl-phenylalanine as the major peptide neutrophil chemotactic factor produced by Escherichia coli. J Biol Chem 1984;259:5430-9.

10. Merlin D, Steel A, Gewirtz AT, Si-Tahar M, Hediger MA, Madara JL. hPepT1mediated epithelial transport of bacteria-derived chemotactic peptides enhances neutrophil-epithelial interactions. J Clin Invest 1998;102:2011-8.

11. Foster DR, Zheng X. Cephalexin inhibits N-formylated peptide transport and intestinal hyperpermeability in Caco2 cells. J Pharm Pharm Sci 2007;10:299-310.

12. Buyse M, Tsocas A, Walker F, Merlin D, Bado A. PepT1-mediated fMLP transport induces intestinal inflammation in vivo. Am J Physiol Cell Physiol 2002;283:C1795-800.

13. Shi B, Song D, Xue H, Li J, Li N, Li J. Abnormal expression of the peptide transporter PepT1 in the colon of massive bowel resection rat: a potential route for colonic mucosa damage by transport of fMLP. Dig Dis Sci 2006;51:2087-93.

14. Baeuerle PA, Henkel T. Function and activation of NF-kappa B in the immune system. Annu Rev Immunol 1994;12:141-79.

15. Sica A, Dorman L, Viggiano V, et al. Interaction of NF-kappaB and NFAT with the interferon-gamma promoter. J Biol Chem 1997;272:30412-20.

16. Buchman AL, Moukarzel AA, Bhuta S, et al. Parenteral nutrition is associated with intestinal morphologic and functional changes in humans. JPEN J Parenter Enteral Nutr 1995;19:453-60.

17. Omata J, Pierre JF, Heneghan AF, et al. Parenteral nutrition suppresses the bactericidal response of the small intestine. Surgery 2013;153:17-24.

18. Heneghan AF, Pierre JF, Tandee K, et al. Parenteral nutrition decreases paneth cell function and intestinal bactericidal activity while increasing susceptibility to bacterial enteroinvasion. JPEN J Parenter Enteral Nutr 2014;38:817-24.

19. Howard A, Goodlad RA, Walters JR, Ford D, Hirst BH. Increased expression of specific intestinal amino acid and peptide transporter mRNA in rats fed by TPN is reversed by GLP-2. J Nutr 2004;134:2957-64. 


\section{Articles | Nosworthy and Brunton}

20. Jones DP. Redefining oxidative stress. Antioxid Redox Signal 2006;8: 1865-79.

21. Nosworthy MG, Dodge ME, Bertolo RF, Brunton JA. Enterally delivered dipeptides improve small intestinal inflammatory status in a piglet model of intestinal resection. Clin Nutr 2015; S0261-5614(15)00145-4.

22. Cianciulli A, Acquafredda A, Cavallo P, et al. f-Met-Leu-Phe stimulates nitric oxide production in chick embryo neurons: the role of NF-kB. Immunopharmacol Immunotoxicol 2009;31:51-63.

23. Browning DD, Pan ZK, Prossnitz ER, Ye RD. Cell type- and developmental stage-specific activation of NF-kappaB by fMet-Leu-Phe in myeloid cells. J Biol Chem 1997;272:7995-8001.

24. Pan ZK, Chen LY, Cochrane CG, Zuraw BL. fMet-Leu-Phe stimulates proinflammatory cytokine gene expression in human peripheral blood monocytes: the role of phosphatidylinositol 3-kinase. J Immunol 2000;164:404-11.

25. Grell M, Douni E, Wajant H, et al. The transmembrane form of tumor necrosis factor is the prime activating ligand of the $80 \mathrm{kDa}$ tumor necrosis factor receptor. Cell 1995;83:793-802.

26. Hasegawa S, Ichiyama T, Sonaka I, et al. Cysteine, histidine and glycine exhibit anti-inflammatory effects in human coronary arterial endothelial cells. Clin Exp Immunol 2012;167:269-74.

27. Inoue Y, Espat NJ, Frohnapple DJ, Epstein H, Copeland EM, Souba WW. Effect of total parenteral nutrition on amino acid and glucose transport by the human small intestine. Ann Surg 1993;217:604-12; discussion 612-4.
28. Fukatsu K, Kudsk KA, Zarzaur BL, Wu Y, Hanna MK, DeWitt RC. TPN decreases IL-4 and IL-10 mRNA expression in lipopolysaccharide stimulated intestinal lamina propria cells but glutamine supplementation preserves the expression. Shock 2001;15:318-22.

29. Sun X, Yang H, Nose K, et al. Decline in intestinal mucosal IL-10 expression and decreased intestinal barrier function in a mouse model of total parenteral nutrition. Am J Physiol Gastrointest Liver Physiol 2008;294:G139-47.

30. Illig KA, Ryan CK, Hardy DJ, Rhodes J, Locke W, Sax HC. Total parenteral nutrition-induced changes in gut mucosal function: atrophy alone is not the issue. Surgery 1992;112:631-7.

31. Iiboshi Y, Nezu R, Kennedy M, et al. Total parenteral nutrition decreases luminal mucous gel and increases permeability of small intestine. JPEN J Parenter Enteral Nutr 1994;18:346-50.

32. Mosenthal AC, Xu D, Deitch EA. Elemental and intravenous total parenteral nutrition diet-induced gut barrier failure is intestinal site specific and can be prevented by feeding nonfermentable fiber. Crit Care Med 2002;30:396-402.

33. Varma TK, Toliver-Kinsky TE, Lin CY, Koutrouvelis AP, Nichols JE, Sherwood ER. Cellular mechanisms that cause suppressed gamma interferon secretion in endotoxin-tolerant mice. Infect Immun 2001;69:5249-63.

34. Dodge ME, Bertolo RF, Brunton JA. Enteral feeding induces early intestinal adaptation in a parenterally fed neonatal piglet model of short bowel syndrome. JPEN J Parenter Enteral Nutr 2012;36:205-12. 